PROCEEDINGS OF THE

AMERICAN MATHEMATICAL SOCIETY

Volume 125, Number 10, October 1997, Pages 2987-2996

S 0002-9939(97)03873-2

\title{
BLOCH-TO-BMOA PULLBACKS ON THE DISK
}

\author{
BOO RIM CHOE, WADE RAMEY, AND DAVID ULLRICH
}

(Communicated by Theodore Gamelin)

\begin{abstract}
For a given holomorphic self map $\varphi$ of the unit disk, we consider the Bloch-to-BMOA composition property (pullback property) of $\varphi$. Our results are (1) $\varphi$ cannot have the pullback property if $\varphi$ touches the boundary too smoothly, (2) while $\varphi$ has the pullback property if $\varphi$ touches the boundary rather sharply. One of these results yields an interesting consequence completely contrary to a higher dimensional result which has been known. These results resemble known results concerning the compactness of composition operators on the Hardy spaces. Some remarks in that direction are included.
\end{abstract}

\section{INTRODUCTION}

We begin with an easy observation. Let $f$ be an $L^{p}$-Bergman function on the unit disk $D$ of the complex plane. That is, let $f$ be an $L^{p}$-holomorphic function on $D$. Then the subharmonicity of $|f|^{p}$ easily yields the radial growth condition

$$
|f(z)|\left(1-|z|^{2}\right)^{2 / p}=O(1)
$$

and therefore we have

$$
\int_{0}^{2 \pi}\left|f\left(\frac{1+e^{i t}}{2}\right)\right|^{q} d t<\infty
$$

for $q<p / 4$. In other words, composition with the function $\varphi(z)=(1+z) / 2$ takes the $L^{p}$-Bergman space $A^{p}$ into the Hardy space $H^{q}$ for $q<p / 4$ (of course, this is not sharp). It follows that composition with $\varphi$ takes $\cap A^{p}$ into $\cap H^{p}$. The next step after this Bergman-to-Hardy composition property is perhaps the analogous Blochto- $B M O A$ composition property. However, one can see from a direct calculation using Proposition 1 below that the Bloch-to- $B M O A$ composition property does not hold for $\varphi$. Motivated by this simple example, we consider the Bloch-to-BMOA composition property in the present paper.

To be more precise, write $\mathcal{B}$ for the Bloch space consisting of holomorphic functions $f$ on $D$ whose derivatives satisfy the radial growth condition

$$
\left|f^{\prime}(z)\right|\left(1-|z|^{2}\right)=O(1)
$$

and let $B M O A$ denote the space of $H^{1}$-functions $f$ whose boundary functions $f$ have bounded mean oscillations with respect to subarcs of the unit circle $T$. These spaces $\mathcal{B}$ and $B M O A$ can be considered as limiting spaces of the Bergman and

Received by the editors September 22, 1995 and, in revised form, May 17, 1996.

1991 Mathematics Subject Classification. Primary 30D45, 47B38.

Key words and phrases. Bloch space, BMOA, pullback property.

The first author is supported in part by BSRI (96-1407) and GARC (96) of Korea. 
Hardy spaces in the sense that $\mathcal{B}$ and $B M O A$ are identified with the dual spaces of $L^{1}$-Bergman space and $H^{1}$, respectively (see [14, Theorems 5.1.4 and 8.3.8]). We will say that a holomorphic self map $\varphi$ of $D$ has the pullback property if it has the following Bloch-to- $B M O A$ composition property:

$$
f \circ \varphi \in B M O A \text { for all } f \in \mathcal{B} .
$$

Note. The pullback property is trivial if $\varphi(D)$ is contained in a compact subset of $D$. So, the function $\varphi$ will always denote a holomorphic self map of $D$ with supremum norm one in the rest of the paper.

Our results of the present paper indicate that the pullback property depends upon how "smoothly" (or how "sharply") a function under consideration touches the boundary. Roughly speaking, a smooth boundary contact even at just one single point forces the failure of the pullback property. On the other hand, a rather sharp boundary contact helps the pullback property. For example, our results imply the following:

- Every function $\varphi \in \Lambda_{1}$ fails to have the pullback property.

- For a given $0<\alpha<1$, there exists $\varphi \in \Lambda_{\alpha}$ for which the pullback property holds.

Here, $\Lambda_{\alpha}$ denotes the holomorphic Lipschitz class of order $\alpha$ consisting of all holomorphic functions $f$ on $D$ such that

$$
|f(z)-f(w)| \leq C|z-w|^{\alpha} \quad(z, w \in D)
$$

for some constant $C=C_{f}$. Note that the pullback property can also be considered for a holomorphic function taking the unit ball $B_{n} \subset \mathbb{C}^{n}$ into $D$. Such higher dimensional pullback property has been studied by several authors ([1], [2], [5], [6], [10], [11], [15]) and the most general known result is ([10]): Every holomorphic Lipschitz function of order 1 taking $B_{n}(n>1)$ into D has the "pullback" property. It is interesting to see that this higher dimensional result is completely contrary to the one dimensional result mentioned above.

In the second section, we use the notion of angular derivatives to quantify the smoothness of boundary contact and show that the existence of an angular derivative even at just one single boundary point implies the failure of the pullback property. Then we show that only finitely many nontangential boundary contacts ensure the pullback property. The proof uses a characterization of BMOA in terms of Carleson measures. At the end of the section, a question suggested by our results is included. In the third section, we compare our results with those concerning compact composition operators on well-known function spaces like the Hardy spaces. In particular, we notice that our results look like the "criterion" for a holomorphic self map of $D$ to induce a compact composition operator on $H^{2}$. Some remarks in that direction are included.

\section{Pullback PRoperty}

As is well-known, a certain Carleson measure condition characterizes the space $B M O A$ : A holomorphic function $f$ on $D$ belongs to $B M O A$ if and only if $\left|f^{\prime}\right|^{2}\left(1-|z|^{2}\right) d A$ is a Carleson measure. Here, $d A$ denotes the area measure on $D$ normalized to have total mass one. More explicitly, let

$$
Q_{\delta}(\zeta)=\{r \eta: \eta \in T, 1-\delta<r<1,|1-\eta \bar{\zeta}|<\delta\}
$$


denote the Carleson square at $\zeta \in T$ with size $\delta>0$. Then, for a holomorphic function $f$ on $D$, we have $f \in B M O A$ if and only if

$$
\sup \frac{1}{\delta} \int_{Q_{\delta}(\zeta)}\left|f^{\prime}\right|^{2}\left(1-|z|^{2}\right) d A<\infty
$$

where sup is taken over all $Q_{\delta}(\zeta)$. See, for example, [8, Theorem 6.3.4] or [14, Theorem 8.3.5]. Using this Carleson measure characterization of $B M O A$, we obtain the following necessary and sufficient condition for $\varphi$ to have the pullback property, which is useful for our purposes.

Proposition 1. The pullback property holds for $\varphi$ if and only if

$$
\sup \frac{1}{\delta} \int_{Q_{\delta}(\zeta)} \frac{\left(1-|z|^{2}\right)\left|\varphi^{\prime}\right|^{2}}{\left(1-|\varphi|^{2}\right)^{2}} d A<\infty
$$

where sup is taken over all $Q_{\delta}(\zeta)$.

Proof. Suppose (2) holds. Then the pullback property of $\varphi$ easily follows from the Carleson measure characterization (1). To prove the converse, pick two Bloch functions $g$ and $h$ such that

$$
\left|g^{\prime}(z)\right|^{2}+\left|h^{\prime}(z)\right|^{2} \geq \frac{1}{\left(1-|z|^{2}\right)^{2}} \quad(z \in D) .
$$

Such Bloch functions are constructed by Ramey and Ullrich [10, Proposition 5.4]. Since $g \circ \varphi, h \circ \varphi \in B M O A$ by assumption, the measure

$$
\left(\left|g^{\prime}(\varphi)\right|^{2}+\left|h^{\prime}(\varphi)\right|^{2}\right)\left|\varphi^{\prime}\right|^{2}\left(1-|z|^{2}\right) d A
$$

is a Carleson measure. Hence (2) follows from the above inequality. The proof is complete.

Our first result is that if $\varphi$ touches a boundary point too smoothly, then $\varphi$ fails to have the pullback property. With this in mind, let us recall the notion of angular derivatives. For a holomorphic function $f: D \rightarrow D$, we say that $f$ has an angular derivative at a point $\zeta \in T$ if $f$ has a unimodular nontangential limit $f(\zeta) \in T$ at $\zeta$ and if the difference quotient

$$
\frac{f(z)-f(\zeta)}{z-\zeta}
$$

has a finite limit as $z \rightarrow \zeta$ nontangentially. This limit, if it exists, is naturally denoted by $f^{\prime}(\zeta)$. The requirement $|f(\zeta)|=1$ may seem peculiar. But its significance lies in the classical Julia-Carathédory theorem (see [4, Sections 298-299]) which enables us to make an interesting connection between the pullback property and angular derivatives.

The Julia-Carathéodory Theorem. Let $f: D \rightarrow D$ be a holomorphic function such that

$$
\liminf \frac{1-|f(z)|^{2}}{1-|z|^{2}}=d<\infty \quad(z \rightarrow \zeta \in T \quad \text { unrestrictedly }) .
$$

Then $f$ has an angular derivative $f^{\prime}(\zeta)=f(\zeta) \bar{\zeta}$ d at $\zeta$. In addition, $f^{\prime}(\zeta)$ is the nontangential limit at $\zeta$ of the complex derivative $f^{\prime}$. 
Note that if (3) holds, then

$$
\frac{1-|f(0)|}{1+|f(0)|} \leq\left|f^{\prime}(\zeta)\right|
$$

by the Schwarz-Pick lemma (see $\left[8\right.$, Lemma 1.1.2]) and thus $f^{\prime}(\zeta) \neq 0$. Thus one can see that $f$ is nontangentially conformal at $\zeta$. More precisely, a curve in $D$ ending at $\zeta$ and making angle $0<\alpha<\pi$ with $T$ is mapped onto a curve ending at $f(\zeta)$ and making the same angle with $T$. In particular, the range of such $f$ must have a "smooth" contact at $\zeta$ with $T$, which is the geometric picture we have at a point where an angular derivative exists.

Theorem 2. If $\varphi$ has an angular derivative at a boundary point, then $\varphi$ fails to have the pullback property.

Proof. Without loss of generality, assume $\varphi(1)=1$ and $\varphi$ has an angular derivative at 1 with $\varphi^{\prime}(1)=d>0$. Then we have $\varphi(z)=1-d(1-z)+o(|1-z|)$ and thus

$$
|\varphi(z)|^{2}=1-2 d \operatorname{Re}(1-z)+o(|1-z|) \quad(z \rightarrow 1 \quad \text { nontangentially }) .
$$

Since $o(|1-z|)=o\left(1-|z|^{2}\right)$ as $z \rightarrow 1$ nontangentially and $\operatorname{Re}(1-z) /\left(1-|z|^{2}\right)$ has nontangential limit $1 / 2$ at 1 , it follows that

$$
\lim \frac{1-|\varphi(z)|^{2}}{1-|z|^{2}}=d \quad(z \rightarrow 1 \quad \text { nontangentially })
$$

and therefore, by the Julia-Carathéodory theorem, we have

$$
\lim \left|\varphi^{\prime}(z)\right|=d \quad(z \rightarrow 1 \quad \text { nontangentially })
$$

For $0<t<1$, let $Q_{\delta, t}$ denote the set of points $z \in Q_{\delta}=Q_{\delta}(1)$ such that $|z|<1-t \delta$. One can verify that

$$
|1-z|<\frac{1-|z|^{2}}{t} \quad\left(z \in Q_{\delta, t}\right)
$$

This means that, for each fixed $t$, all $Q_{\delta, t}$ are contained in a common nontangential approach region at 1 . Thus it follows from (4), (5) that

$$
\lim _{\delta \rightarrow 0} \frac{1}{\delta} \int_{Q_{\delta, t}} \frac{\left|\varphi^{\prime}\right|^{2}\left(1-|z|^{2}\right)}{\left(1-|\varphi|^{2}\right)^{2}} d A=\lim _{\delta \rightarrow 0} \frac{1}{\delta} \int_{Q_{\delta, t}} \frac{d A}{1-|z|^{2}}=\frac{1}{\pi} \log \frac{1}{t} .
$$

Now, letting $t \searrow 0$, one finds that condition (2) is violated. The proof is complete.

Note that every $\varphi \in \Lambda_{1}$ has angular derivatives at points $\zeta \in T$ where $|\varphi(\zeta)|=1$ by the Julia-Carathéodory theorem. Thus we have

Corollary 3. Every $\varphi \in \Lambda_{1}$ fails to have the pullback property.

There is another interesting condition which implies the existence of an angular derivative. As is well known (see, for example, [3]), a holomorphic self map of $D$ with no fixed point in $D$ has an angular derivative (whose absolute value is at most one) at its unique Denjoy-Wolff point. This yields

Corollary 4. If $\varphi$ has the pullback property, then $\varphi$ has a fixed point in D.

Some other geometric conditions that imply the existence of an angular derivative can be found in [13]. 
Remark. For a given $\varphi$, let $M_{\varphi}=\left\{\zeta \in T: \sup _{0<r<1}|\varphi(r \zeta)|=1\right\}$. Thus $M_{\varphi}$ is the set of all points where $\varphi$ touches the boundary. As indicated in [2], we must have $\sigma\left(M_{\varphi}\right)=0$ where $\sigma$ denotes the arclength measure on $T$ normalized to have total mass one. It is known ([9, Example 3.6]) that there exists a nonconstant inner function having no angular derivative. Hence, the converse of Theorem 2 is false.

Our next result is that $\varphi$ has the pullback property in case $\varphi$ touches the boundary only at finitely many points and all contacts are nontangential. Note that such a function $\varphi$ takes $D$ into some polygon inscribed in $T$. We first prove a lemma, which is particularly useful when one wishes to prove the pullback property of a given function whose range is contained in some simply connected region such as a polygon.

Lemma 5. If $\varphi$ has the pullback property, then so does $\varphi \circ g$ for every holomorphic self map $g$ of $D$.

Proof. It is sufficient to show that $B M O A$ is invariant under composition with a given holomorphic self map. This follows from the well-known theorem (see [14, Theorem 8.3.11]) that a holomorphic function $f$ on $D$ belongs to $B M O A$ if and only if there exists some bounded harmonic function $u$ such that $f=u+\tilde{u}$ where $\tilde{u}$ denotes the harmonic conjugate function of $u$. The proof is complete.

Theorem 6. If $\varphi$ takes $D$ into a polygon inscribed in $T$, then $\varphi$ has the pullback property.

Proof. We may assume by Lemma 5 that $\varphi$ is a Riemann mapping that takes $D$ univalently onto some polygon $P$ inscribed in $T$. By Proposition 2 and the SchwarzPick lemma it is sufficient to show that

$$
\int_{Q_{\delta}(\zeta)} \frac{\left|\varphi^{\prime}\right|}{1-|\varphi|^{2}} d A=O(\delta)
$$

uniformly in $\zeta \in T$. Given a vertex $v$ of $P$, let $v^{*} \in T$ be a point such that $\varphi\left(v^{*}\right)=v$. Here we used the fact that $\varphi$ extends to a homeomorphism of $\bar{D}$ onto $\bar{P}$. Then, a reflection argument shows that there are a closed disk $N_{v}$ with center at $v^{*}$, a non-vanishing holomorphic function $h$ on that disk, and an exponent $0<\alpha(v)<1$ such that

$$
v-\varphi(z)=\left(v^{*}-z\right)^{\alpha(v)} h(z) \quad\left(z \in N_{v}\right) .
$$

This implies

$$
\left|\varphi^{\prime}(z)\right| \leq C\left|v^{*}-z\right|^{\alpha(v)-1} \quad\left(z \in N_{v}\right)
$$

Here, and in the rest of the proof, $C$ denotes various constants, depending only on the function $\varphi$, whose values may change with each occurrence. Note that $\varphi$ maps $D \cap N_{v}$ into a nontangential region with vertex at $v$. More explicitly, we have

$$
1-|\varphi(z)|^{2} \geq C|v-\varphi(z)| \geq C\left|v^{*}-z\right|^{\alpha(v)}
$$

for $z \in D \cap N_{v}$.

To estimate the integral in the left side of (6), it is clearly enough to consider $\delta$ sufficiently small so that $Q_{3 \delta}\left(v^{*}\right) \subset N_{v}$ for all vertices $v$ of $P$. Let $\zeta \in T$. We first consider the case where $\left|\zeta-v^{*}\right|<2 \delta$ for some vertex $v$. In this case, we have 
$Q_{\delta}(\zeta) \subset Q_{3 \delta}\left(v^{*}\right) \subset N_{v}$. It follows from (7) and (8) that the integral in the left side of $(6)$ is dominated by

$$
C \int_{Q_{3 \delta}\left(v^{*}\right)} \frac{d A}{\left|v^{*}-z\right|}=O(\delta) .
$$

Next, consider the case where $\left|\zeta-v^{*}\right| \geq 2 \delta$ for all vertices $v$. In this case $\left|v^{*}-z\right| \geq \delta$ for $z \in Q_{\delta}(\zeta)$. If, in addition, $z \in N_{v}$ for some vertex $v$, then we see from (7) and (8) that

$$
\frac{\left|\varphi^{\prime}(z)\right|}{1-|\varphi(z)|^{2}} \leq \frac{C}{\delta}
$$

Since $\left|\varphi^{\prime}\right|\left(1-|\varphi|^{2}\right)^{-1}$ is bounded on the complement in $D$ of the union of disks $N_{v}$, the above holds for all $z \in Q_{\delta}(\zeta)$. Consequently, the integral in the left side of (6) is dominated by $C \delta^{-1} A\left(Q_{\delta}(\zeta)\right)=O(\delta)$. This completes the proof.

A theorem of Hardy and Littlewood (see [7, Theorem 5.1]) characterizes functions $f$ in $\Lambda_{\alpha}$ by the continuous extension property up to the boundary and the radial growth condition

$$
\left|f^{\prime}(z)\right|\left(1-|z|^{2}\right)^{1-\alpha}=O(1) .
$$

It is implicit in the proof of Theorem 6 that the derivative of a Riemann mapping $\varphi$ taking $D$ univalently onto a polygon $P$ inscribed in $T$ satisfies the radial growth condition $\left|\varphi^{\prime}(z)\right|\left(1-|z|^{2}\right)^{1-\alpha}=O(1)$ where $\alpha$ is the smallest vertex angle of $P$ divided by $\pi$ and thus $\varphi \in \Lambda_{\alpha}$ by the Hardy-Littlewood theorem mentioned above. This yields

Corollary 7. For a given $0<\alpha<1$, there exists $\varphi \in \Lambda_{\alpha}$ for which the pullback property holds.

We close this section with a question suggested by Theorem 6 .

Question. Consider a region $R$ that is the union of nontangential approach regions with vertices in a countable subset of $T$, or even in a subset of $T$ having arclength measure zero. When does a map taking $D$ onto $R$ have the pullback property?

\section{Related REMARKS}

Let $\mathcal{D}$ denote the Dirichlet space consisting of holomorphic functions on $D$ whose derivatives are square integrable. It is easy to see from (1) that $\mathcal{D}$ is contained in $B M O A$ (in fact in $V M O A$ ). Also, by a similar argument to the proof of Proposition 1 , one can easily verify that composition with $\varphi$ takes $\mathcal{B}$ into $\mathcal{D}$ if and only if

$$
\int_{D} \frac{\left|\varphi^{\prime}\right|^{2}}{\left(1-|\varphi|^{2}\right)^{2}} d A<\infty
$$

It is also easy to see that $\varphi$ induces a Hilbert-Schimidt operator on $\mathcal{D}$ if and only if (9) holds ( [13, Proposition 3.4]). Thus it follows from [9, Theorem 5.3] that (9) is a sufficient condition for $\varphi$ to induce a compact composition operator on $H^{2}$. Note that the integral in the left side of (2) is dominated by $2 \delta$ times the integral in the left side of (9). Thus (9) is also a sufficient condition for $\varphi$ to have the pullback property. 
Also, it has been known ( [13, Theorem 5.1]) that any holomorphic function taking $D$ into a polygon inscribed in $T$ induces a compact composition operator on $H^{2}$. On the other hand ( [13, Theorem 2.1]), functions having an angular derivative fail to induce compact composition operators on $H^{2}$. Thus results on the pullback property and the compact composition operator on $H^{2}$ are very similar in some aspects. So, one may guess that there would exist an implication between the compact composition operator on $H^{2}$ and the pullback property. One implication is true in a certain case:

Proposition 8. If a bounded valent holomorphic self map of D has the pullback property, then it induces a compact composition operator on $H^{2}$.

Proof. This follows from Theorem 2 and the angular derivative criterion of MacCluer and Shapiro (see [9, Theorem 3.10]) asserting that a bounded valent holomorphic self map of $D$ induces a compact composition operator on $H^{2}$ if and only if it fails to have any angular derivative.

We do not know whether the same is true without the bounded valence hypothesis (the answer might be probably no, but we do not have an example). However, we will see that the other implication is false even for univalent functions. In order to see such an example, we first prove

Proposition 9. Composition with a given $\varphi$ takes $\mathcal{B}$ into $H^{2}$ if and only if $\varphi$ is not an extreme point of the unit ball of $H^{\infty}$.

As is well known (see [7, Theorem 7.9]), $\varphi$ is an extreme point of the unit ball of $H^{\infty}$ if and only if

$$
\int_{T} \log \left(1-|\varphi|^{2}\right) d \sigma=-\infty
$$

We remark in passing that a holomorphic self map of $D$ induces a Hilbert-Schmidt composition operator from $\mathcal{D}$ into $H^{2}$ if and only if it is not an extreme point of the unit ball of $H^{\infty}$, which is easily seen from the Littlewood-Paley identity.

Proof. By the Littlewood-Paley identity (see [8, Lemma 6.3.1] or [14, Theorem 8.1.9]), we have

$$
\sup _{0<r<1} \int_{T}|f(r \zeta)|^{2} d \sigma(\zeta)=\int_{D}\left|f^{\prime}\right|^{2} \log \frac{1}{|z|^{2}} d A+|f(0)|^{2}
$$

for holomorphic functions $f$ on $D$. Thus, by using Bloch functions as in the proof of Proposition 1, one can easily verify that composition with $\varphi$ takes $\mathcal{B}$ into $H^{2}$ if and only if

$$
\int_{D} \frac{\left|\varphi^{\prime}\right|^{2}}{\left(1-|\varphi|^{2}\right)^{2}} \log \frac{1}{|z|^{2}} d A<\infty
$$


Now, going backwards to the integral on the boundary by using the LittlewoodPaley identity, one finds that the left side of the above is equal to

$$
\begin{aligned}
& \sum_{n=1}^{\infty} n \int_{D}\left|\varphi^{\prime}\right|^{2}|\varphi|^{2 n-2} \log \frac{1}{|z|^{2}} d A \\
= & \sum_{n=1}^{\infty} \frac{1}{n} \int_{D}\left|\left(\varphi^{n}\right)^{\prime}\right|^{2} \log \frac{1}{|z|^{2}} d A \\
= & \sum_{n=1}^{\infty} \frac{1}{n}\left\{\int_{T}|\varphi|^{2 n} d \sigma-|\varphi(0)|^{2 n}\right\} \\
= & \int_{T} \log \frac{1-|\varphi(0)|^{2}}{1-|\varphi|^{2}} d \sigma .
\end{aligned}
$$

The proof is complete.

The following proposition shows that the pullback analogue of the angular derivative criterion of MacCluer and Shapiro mentioned in the proof of Proposition 8 is false. In particular, the converse of Theorem 2 is false even for univalent functions.

Proposition 10. There exists a univalent function $\varphi$ such that composition with $\varphi$ does not take $\mathcal{B}$ into $H^{2}$ (thus fails to have the pullback property) and yet induces a compact composition operator on $\mathrm{H}^{2}$.

Proof. By Proposition 9, we need to exhibit a univalent function $\varphi$ which is an extreme point of the unit ball of $H^{\infty}$ and yet induces a compact composition operator on $H^{2}$. Such a function is constructed by Shapiro and Sundberg [12, Theorem 3.5].

Now identify constant functions in $\mathcal{B}$ and consider $\mathcal{B}$ as a Banach space with norm

$$
\|f\|_{\mathcal{B}}=\sup _{z \in D}\left|f^{\prime}(z)\right|\left(1-|z|^{2}\right) .
$$

A close look at the proof of Proposition 9 then shows that if $\varphi$ is not an extreme point of the unit ball of $H^{\infty}$, then the operator $C_{\varphi}: \mathcal{B} \rightarrow H^{2}$ defined by

$$
C_{\varphi} f=f \circ \varphi-f(\varphi(0))
$$

is bounded and the operator norm satisfies

$$
\left\|C_{\varphi}\right\|^{2} \leq \int_{T} \log \frac{1-|\varphi(0)|^{2}}{1-|\varphi|^{2}} d \sigma \leq C|| C_{\varphi} \|^{2}
$$

for some constant $C$ independent of $\varphi$. It may be of some independent interest to note that operators $C_{\varphi}$ are uniformly bounded for a certain class of polynomials $\varphi$. Given a positive integer $N$, let $\mathcal{P}_{N}$ denote the class of all holomorphic polynomials $\varphi$, nonvanishing on $D$, of degree less than or equal to $N$. Then the following holds.

Proposition 11. For a given positive integer $N$, operators $C_{\varphi}, \varphi \in \mathcal{P}_{N}$, are uniformly bounded, or equivalently,

$$
\sup _{\varphi \in \mathcal{P}_{N}} \int_{T} \log \frac{1-|\varphi(0)|^{2}}{1-|\varphi|^{2}} d \sigma<\infty
$$


Proof. Let $\varphi \in \mathcal{P}_{N}$. Then $1-|\varphi|^{2}=|f|$ on $T$ for some holomorphic polynomial $f$ of degree less than or equal to $2 N$. The minimum modulus theorem shows that $1-|\varphi(0)|^{2} \leq\|f\|_{\infty}$, the supremum norm of $f$ on $D$. Since

$$
\int_{T} \log |\zeta-a| d \sigma(\zeta)=\log ^{+}|a| \geq \log (1+|a|)-\log 2
$$

for each complex number $a$, we have

$$
\int_{T} \log |f| d \sigma \geq \log \|f\|_{\infty}-m \log 2
$$

where $m$ denotes the degree of $f$. Thus we have

$$
\log \left(1-|\varphi(0)|^{2}\right) \leq \int_{T} \log \left(1-|\varphi|^{2}\right) d \sigma+2 N \log 2 .
$$

This proves the proposition.

As a consequence of Proposition 11, we obtain a BMO type characterization of $\mathcal{B}$. For a similar characterization, see [10].

Corollary 12. For a given positive integer $N$, there exists a constant $C=C_{N}$ such that

$$
C^{-1}\|f\|_{\mathcal{B}}^{2} \leq \sup _{\varphi \in \mathcal{P}_{N}} \int_{T}|f \circ \varphi-f(\varphi(0))|^{2} d \sigma \leq C\|f\|_{\mathcal{B}}^{2}
$$

for all $f \in \mathcal{B}$.

Proof. Considering functions of the form $\varphi(z)=a+(1-|a|) z, 1 / 2<|a|<1$, one obtains the first inequality and the second one follows from Proposition 11 . The proof is complete.

\section{REFERENCES}

[1] P. Ahern, On the behavior near torus of functions holomorphic in the ball, Pacific J. Math. 107 (1983), 267-278. MR 84i:32023

[2] P. Ahern and W. Rudin, Bloch functions, BMO and boundary zeros, Indiana Univ. Math. J. 36 (1987), 131-148. MR 88d:42036

[3] R. B. Burckel, Iterating analytic self maps of the disc, Amer. Math. Monthly 88 (1981), 396-407. MR 82g:30046

[4] C. Carathéodory, Theory of functions of a complex variable, Vol. II, 2nd English edition, Chelsea, New York, 1960. MR 16:346c

[5] J. S. Choa and B. R. Choe, Composition with a homogeneous polynomial, Bull. Korean Math. Soc. 29 (1992), 57-63. MR 94d:32008

[6] B. R. Choe, Cauchy integral equalities and applications, Trans. Amer. Math. Soc. 315 (1989), 337-352. MR 89m:32010

[7] P. L. Duren, Theory of $H^{p}$ spaces, Academic, New York, 1970. MR 42:3552

[8] J. Garnett, Bounded analytic functions, Academic, New York, 1981. MR 83g:30037

[9] B. D. MacCluer and J. H. Shapiro, Angular derivatives and compact composition operators on the Hardy and Bergman spaces, Canad. J. Math. 38 (1986), 878-906. MR 87h:47048

[10] W. Ramey and D. Ullrich, Bounded mean oscillations of Bloch pull-backs, Math. Ann. 291 (1991), 590-606. MR 92i:32004

[11] P. A. Russo, Boundary behavior of $B M O\left(B_{n}\right)$, Trans. Amer. Math. Soc. 292 (1985), 733740. MR 87d:32030

[12] J. H. Shapiro and C. Sundberg, Isolation amongst the composition operators, Pacific J. Math. 145 (1990), 117-152. MR 92g:47041

[13] J. H. Shapiro and P. D. Taylor, Compact, nuclear, and Hilbert-Schmidt composition operators on $H^{2}$, Indiana Univ. Math. J. 23 (1973), 471-496. MR 48:4816 
[14] K. Zhu, Operator theory in function spaces, Dekker, New York, 1990. MR 92c:47031

[15] C. S. Stanton, $H^{p}$ and BMOA pullback properties of smooth maps, Indiana Univ. Math. J. 40 (1991), 1251-1265. MR 93c:32006

Department of Mathematics, Korea University, Seoul, Korea

E-mail address: choebr@semi.korea.ac.kr

Department of Mathematics, Michigan State University, East Lansing, Michigan

E-mail address: ramey@math.msu.edu

Department of Mathematics, Oklahoma State University, Stillwater, Oklahoma

E-mail address: ullrich@hardy.math.okstate.edu 